\title{
In Situ Microscopy Study of ZnO Acid Etching Nanostructures
}

Fangyuan Liu ${ }^{1}$, Xingxu Lu ${ }^{2}$, Zichao Bian ${ }^{2}$, Xiaohui Song ${ }^{2}$, Guoan Zheng ${ }^{2}$ and Pu-Xian Gao ${ }^{2}$

${ }^{1}$ University of Connecticut, Vernon Rockville, Connecticut, United States, ${ }^{2}$ University of Connecticut, Storrs, Connecticut, United States

$\mathrm{ZnO}$ nanostructure has been one of the most popular nanomaterials being studied in the past decades due to its widespread potential applications in photovoltaics, catalysis, flexible electronics, and solid state lighting, etc. [1,2]. In micromachining and nanoelectronic field, etching is an important method to selectively construct two-dimensional or three-dimensional nanostructure semiconductors by introducing etchants or sacrificing the surface with powerful force [3]. To improve the $\mathrm{ZnO}$ nanostructure based device performance, some research groups used acidic or basic wet etching to help to expose the high surface energy crystal planes such as the semipolar $\{101\}$ family surfaces [4]. However, it is hard to control the surface exposure regularity of the etched $\mathrm{ZnO}$ nanostructures.

In this work, the in situ optical microscope is employed to help resolve the crystal shape change in the acid etching process and reveal the morphology transformation process. To clarify the exposed crystal facets after etching, transmission electron microscope (TEM) and scanning electron microscope (SEM) have been used in combination with the optical microscope (OM) observation. By using such a combination method, the etching process of $\mathrm{ZnO}$ nanostructures can be robustly and confidently analyzed from atomic to nanometer scales and the etching mechanism can be simulated.

In order to prepare the sample which can be observed by the optical microscope, the high concentration $(100 \mathrm{mM})$ zinc acetate and HMTA are used for synthesizing the ZnO NAs. SEM image (Figure 1a) shows the diameter of these $\mathrm{ZnO}$ microrods is around $1.4 \sim 2 \mu \mathrm{m}$. $\mathrm{ZnO}$ nanoarrays are reacted with $\mathrm{pH}=2$ hydrochloride acid $(\mathrm{HCl})$ in a continuous flow system at a flow rate of $200 \mu \mathrm{L} / \mathrm{min}$ for $5 \mathrm{~min}$. Figure $\mathbf{1 b}$ indicates that the uniform pencil shape $\mathrm{ZnO}$ has been formed after the etching process. The volume of $\mathrm{ZnO}$ decreases, and the diameter is $0.5 \sim 1 \mu \mathrm{m}$. More new high energy facets are exposed.

The in situ etching process was video-recorded by an Olympus CKX41 and Chameleon®3 Monochrome Camera, for which the time-resolved snapshots and analyzed data were shown in Figures 1c and 1d, respectively. In the beginning, we can clearly observe that the hexagonal structure. After several seconds when the $\mathrm{ZnO} \mathrm{NAs}$ react with $\mathrm{HCl}$, the nanorods are etched by $\mathrm{HCl}$ from the edge and the corner of the region to the center simultaneously. After $60 \mathrm{~s}$, the high contrast surface is smaller than the dark area, which indicates a prism structure is etched. It is attributed to the $\mathrm{HCl}$ firstly etches the top surface $\{001\}$ and the side edges $\{100\}$ of $\mathrm{ZnO}$.

The etched $\mathrm{ZnO}$ nanorod was captured by TEM (Figure 2). The pencil shape ZnO matches the SEM images. Zooming in the tip of this etched $\mathrm{ZnO}$ nanorod, the high-resolution TEM images show that lots of new facets are exposed after hydrochloride etching, and most of them are projected to be $\{101\}$ surfaces, which enclose the pencil shaped structure after etching.

In summary, $\mathrm{ZnO}$ nanoarrays are etched to construct different morphology by acid. In situ OM, TEM and SEM are combined to reveal this etching process. The beam effect and resolution limitation could be settled, which was beneficial to restoring the real scene without interfering factors. Large scale $\mathrm{ZnO}$ nanostructures with high surface energy planes are prepared, which can be used as gas sensors, gas catalysts, photovoltaic devices, photonic crystals, etc. 
Acknowledgment: We acknowledge financial support from the US DOE (Award No. DE-EE0008423), the US NSF (Award No. CBET1344792), and the University of Connecticut. The microscopy studies were performed using the facilities in the UConn/Thermo Fisher Scientific Center for Advanced Microscopy and Materials Analysis (CAMMA).
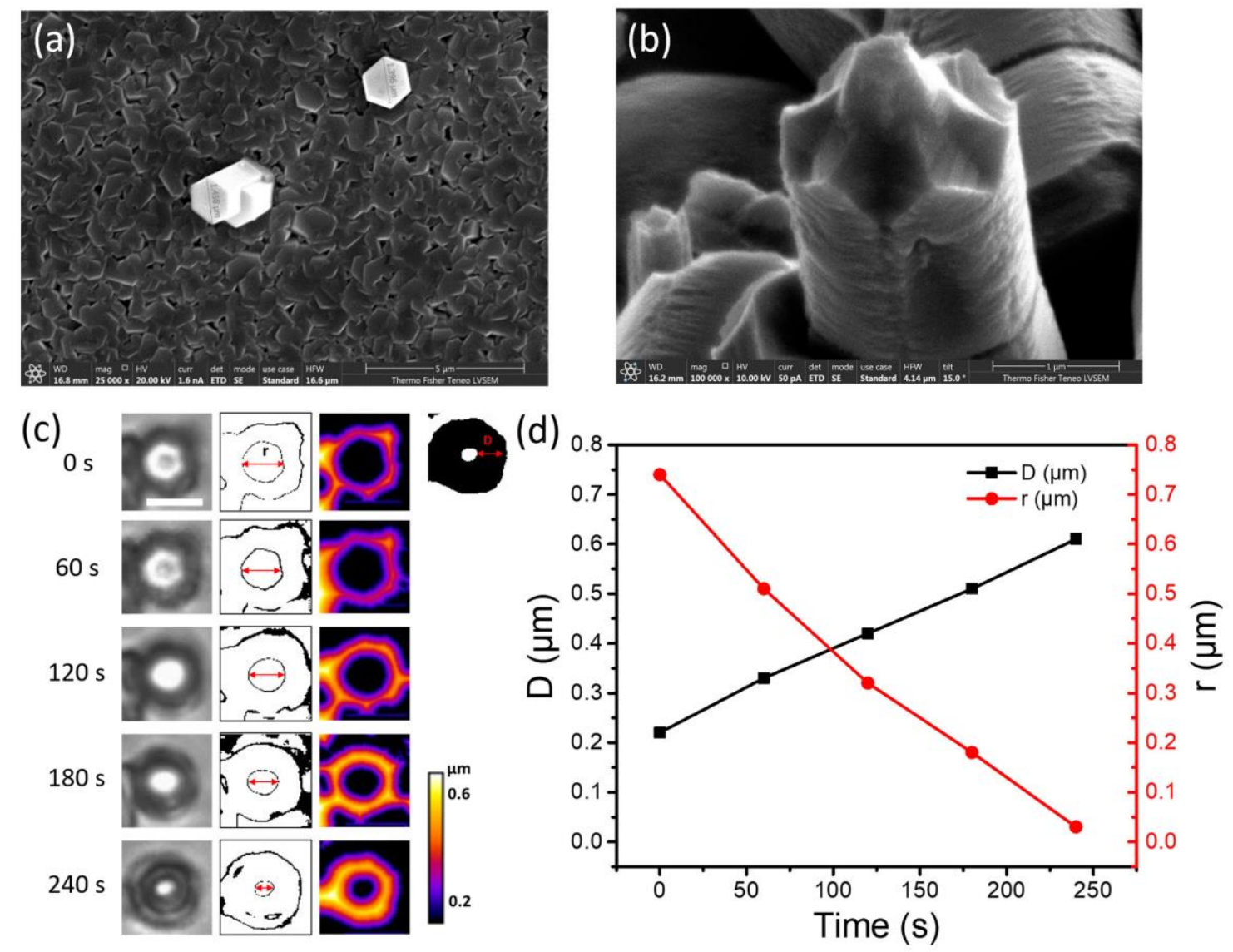

Figure 1. (a) SEM image of wurtzite $\mathrm{ZnO}$ nanorods. (b) $\mathrm{ZnO}$ pencil shape structure after $\mathrm{HCl}$ etching. (c) Optical microscope images of $\mathrm{ZnO}$ nanorod morphology evolution in acid solution. Scale bar: $1 \mathrm{~mm}$. (d) Quantification of $\mathrm{ZnO}$ nanorod size evolution. 


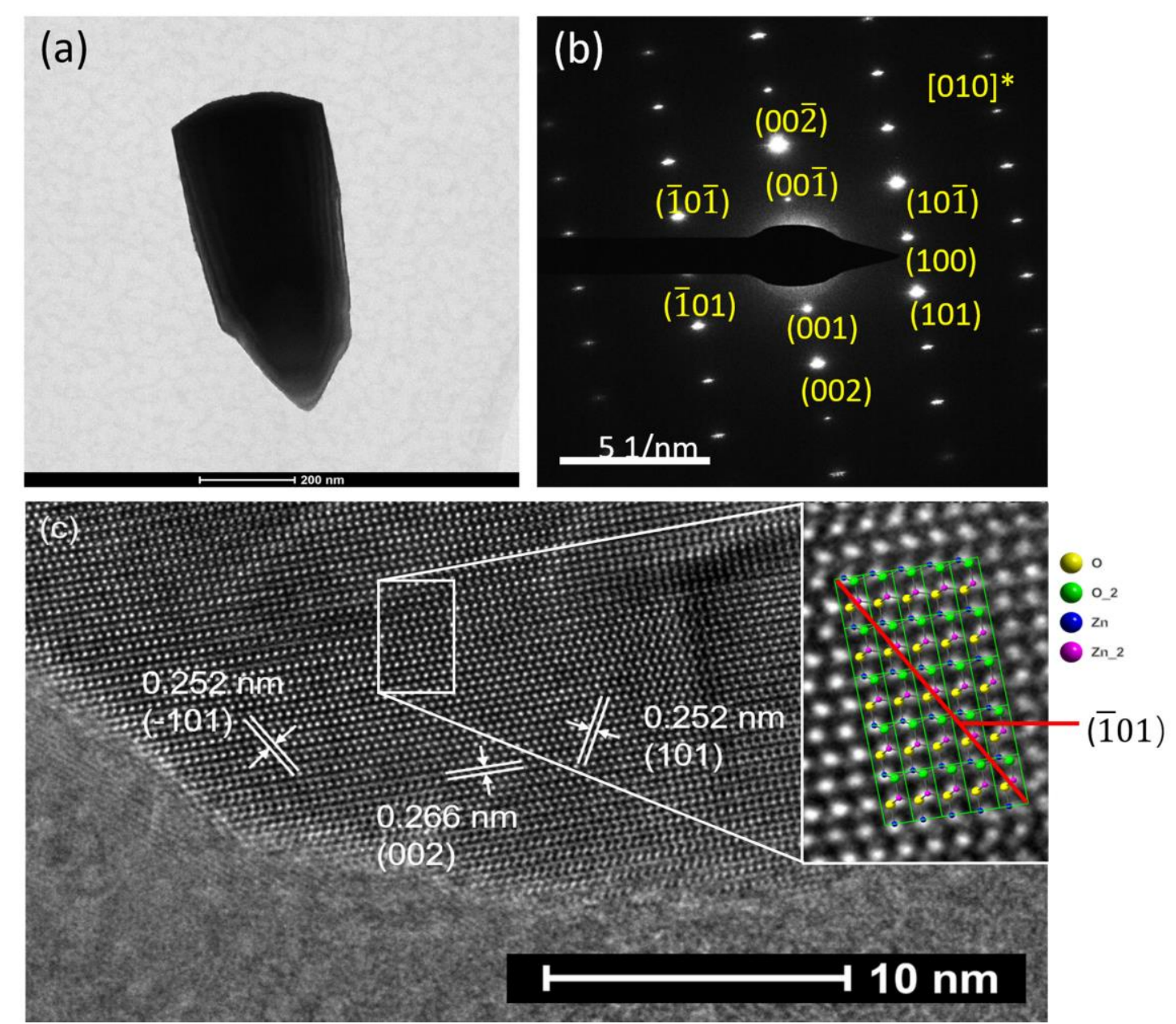

Figure 2. (a) TEM image of etched $\mathrm{ZnO}$ nanorod and (b) corresponding diffraction pattern. (c) HRTEM image of the tip of pencil shape $\mathrm{ZnO}$ nanorod in (a). The insert of (c) is a simulate structure of $\mathrm{ZnO}$.

\section{References}

[1] C.B. Ong, L.Y. Ng, A.W. Mohammad, Renew. Sust. Energ. Rev. 81 (2018), p. 536.

[2] R. Kumar, O. Al-Dossary, G. Kumar, A. Umar, Nano-Micro Lett. 7 (2015), p. 97.

[3] P. Pal, K. Sato, Micro and Nano Systems Lett. 3 (2015), p. 6.

[4] A. Ostapenko, T. Klöffel, J. Eußner, K. Harms, S. Dehnen, B. Meyer, G. Witte, ACS Appl. Mater. Interfaces 8 (2016), p. 13472. 